\title{
Electro-Mechanics in Biventricular Models
}

\author{
FB Sachse ${ }^{1}$, G Seemann², MB Mohr ${ }^{2}$ \\ ${ }^{1}$ Nora Eccles Harrison Cardiovascular Research and Training Institute, University of Utah, USA \\ ${ }^{2}$ Institut für Biomedizinische Technik, Universität Karlsruhe (TH), Germany
}

\begin{abstract}
Cardiac electro-mechanical models are valuable tools to gain insights in physiology and pathophysiology of the heart. Progressive models can be created by fusion of various basic models. In this work biventricular models of cardiac electro-mechanics were developed by fusion of anatomical, electrical, and mechanical models. The importance of anatomical modeling was researched by inclusion of two different anatomical models, i.e. an analytical and a magnetic resonance diffusion tensor imaging based model. The fused models were applied in simulations of physiological behavior and results of these were analyzed. Significant difference of deformation were found, which can be attributed to the anatomical models. The analysis emphasized the importance of appropriate anatomical modeling for simulations of cardiac mechanics.
\end{abstract}

\section{Introduction}

Models of cardiac electro-mechanics can provide a quantitative description of measurement data and improve understanding of physiology and pathophysiology of the heart. Furthermore, predictions of cardiac behavior are possible, e.g. the influence of pathologies to electrophysiology and mechanics.

Fusion of anatomical, electrical, and mechanical models allows simulation of biventricular electro-mechanics. An anatomical model describes the distribution of tissues and macroscopic characteristics of microstructure in left and right ventricle. An electrophysiological model of a ventricular myocyte reconstructs intra- and extracellular ion concentrations, ion flows through the cell membrane and gap junctions, and the transmembrane voltage. The combination with a model of intercellular current flow allows the reconstruction of electrical excitation propagation. Further models describe the development of tension in the sarcomere taking cellular electro-mechanical conditions into account and the deformation of the myocardium under the influence of tension.

In this work three-dimensional hybrid models of ventricles were developed, which allow coupled simulation of electrical excitation propagation, tension development and deformation. Two different anatomical biventricular models were applied: an analytical and a magnetic resonance diffusion tensor imaging (MR DTI) based model. Both models included a locally varying orientation of myocytes, which leads to anisotropy of electrical and mechanical properties. Further components of the hybrid model were an adapted Noble-VargheseKohl-Noble model of electrophysiology of a ventricular myocyte, an adapted Glaenzel-Sachse-Seemann model of tension development, and a continuum mechanics based deformation model applying the material law of Guccione-McCulloch-Waldman. The intercellular current flow was calculated with the monodomain model. The monodomain model applied the generalized Poisson's equation for electrical current fields, which is discretized on deformable grids with the finite element method. The tension development was controlled by the concentration of intracellular calcium and fiber strain. Deformation was calculated using an incremental Lagrangian formulation with displacement-based isoparametric finite elements.

\section{Methods}

\subsection{Analytical biventricular model}

A small mammal's left and right ventricle were approximated by an analytical model. Both ventricles were described by crop of confocal truncated ellipsoids of proper size and shape. The ventricular fiber orientation was interpolated from boundary conditions reflecting knowledge from anatomical studies [1]. The subepicardial orientation was set to $-75^{\circ}$, midwall to $0^{\circ}$, and subendocardial to $75^{\circ}$.

The applied analytical model consisted of 40 x 30 x 48 cubic voxels. The number of voxels was reduced by 2 in each dimension for creating a model of passive mechanics.

\subsection{MR DTI biventricular model}

A biventricular model was constructed with image processing based strategies as detailed in a previous report [2]. In short, a canine heart was ex-planted, fixed and imaged using proton density (PD) and diffusion tensor imaging. The imaging was done at the Center for In Vivo 
Microscopy, Duke University, Durham (USA). The images were stored in three dimensional data sets, consisting of $256 \times 128 \times 128$ voxels with a size of $0.4 \mathrm{~mm} \times 0.8 \mathrm{~mm}$ x $0.6 \mathrm{~mm}$. The PD of each voxel was coded by a scalar value, the diffusion tensor by its three principal axes and three eigenvalues.

The images were pre-processed to simplify the subsequent processes. Segmentation and classification of the data sets were performed using techniques of digital image processing, e.g. interactively deformable meshes, thresholding, region growing, and morphological operators [3]. The boundaries of subepi- and -endocardial as well as septal myocardium were determined using interactively deformable triangle meshes [4]. Suitable initial meshes were manually placed and subsequently deformed to fit with tissue boundaries. The space enclosed by the meshes served as a mask for subsequent refining by thresholding. Region growing was used to classify blood, papillary muscles, left and right ventricular myocardium. Sequences of morphological operators, i.e. median filtering, opening and closing, eliminated minor fail assignments.

In this work a simplified MR DTI model was applied comprising $128 \times 128 \times 96$ cubic voxels. The model was reduced to $32 \times 32 \times 24$ voxels for describing passive mechanics.

\subsection{Modeling of cellular electrophysiology}

The Noble-Varghese-Kohl-Noble model describes the electrophysiology and tension development of a ventricular myocyte of guinea-pig. The model includes effects on ionic channels by the concentration of adenosine triphosphate (ATP) and acetylcholine (ACh) as well as by stretching. A description of the diadic space is incorporated. Different variants and configurations of the model exist. The variant applied in this work is based on [5] using only the electrophysiological part of the model.

\subsection{Modeling of excitation propagation}

Different modeling approaches of the electrical excitation propagation in the myocardium can be distinguished depending on the representation of the microscopic and macroscopic anatomy as well as depending on the approximation of the cellular electrophysiology [7, 6]. In this work a monodomain model was used, which describes the electrical current flow in the intracellular domain by the generalized Poisson's equation. The hereby arising 2nd order differential equations were discretized and solved on deformable grids with the finite element method. A deformation dependent transformation of the intracellular conductivity tensor was performed [6].

\subsection{Modeling of tension development}

Glaenzel et al. described a model reproducing the tension development in cardiac muscle [8, 9], which combines a description of the binding of intracellular calcium to troponin (TN) $\mathrm{C}$, the configuration change of tropomyosin (TM), and the interaction of actin and myosin, particularly the formation of cross-bridges (XB). The calcium binding to TN $\mathrm{C}$ is similarly described as in the 3rd Rice-Winslow-Hunter model [10]. The interaction of actin and myosin is adopted from Gordon et al. [11], Bers et al. [12], and Spudich [13]. The cooperativity mechanisms XB-TN, XB-XB, and TM-TM are incorporated in the tension development model. The model uses as input the concentration of intracellular calcium $\left[\mathrm{Ca}^{2+}\right]_{i}$, the sarcomere stretch $\lambda$, and the sarcomere stretch velocity $v$. The model delivers as output a normalized tension.

\subsection{Elastomechanical modeling}

Various approaches exist to model passive mechanics of biological tissue. The approaches can be classified concerning description of material properties, representation of spatial domain, and numerical aspects. Commonly, material properties, i.e. the relationship between stress and strain, are assessed by uni-, bi- and triaxial measurements of specimens. Mechanical properties of biological tissue are found to be nonlinear, anisotropic, and viscoelastic. The properties can be described by e.g. strain energy density functions. The spatial domain can be represented with point, surface, and volume elements. Finite differences techniques, e.g. spring-mass systems [14], can be differentiated from boundary [15] and finite element techniques [16].

In this work a deformation model was applied, which bases on the strain energy density function of GuccioneMcCulloch-Waldman for passive ventricular myocardium of canine [17]. The calculation of deformation was performed in an incremental Lagrangian formulation with displacement-based isoparametric finite elements $[6,16]$.

\section{Results}

The properties of the models were investigated by exemplary simulations. In these simulations the positions in the valve plane $(z=0)$ were fixed. The simulations started by applying an electrical stimulus at the apex at time $t=0 \mathrm{~ms}$ and had a duration of $500 \mathrm{~ms}$. The displacements were determined every $2.5 \mathrm{~ms}$. Every $20 \mu s$ a calculation of each voxel was performed in the electrophysiological, excitation and tension development model.

Results of these simulation are shown in figures 1,2 and 3 . The simulations with the hybrid models showed a rapid spread of electrical excitation from the stimulus site to basal, a delayed spread of increased intracellular calcium concentration, and a further delayed spread of tension development. The analytical model showed significant rotation and basal movement of the apex. The MR DTI 


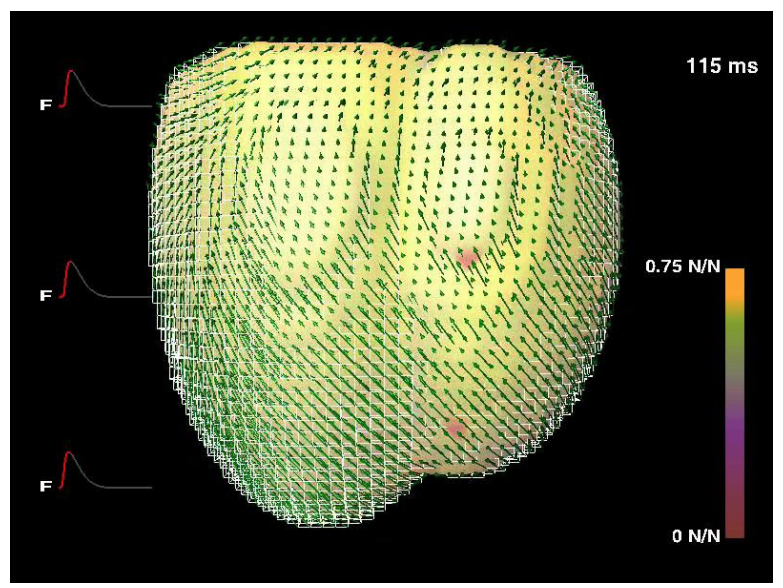

(a)

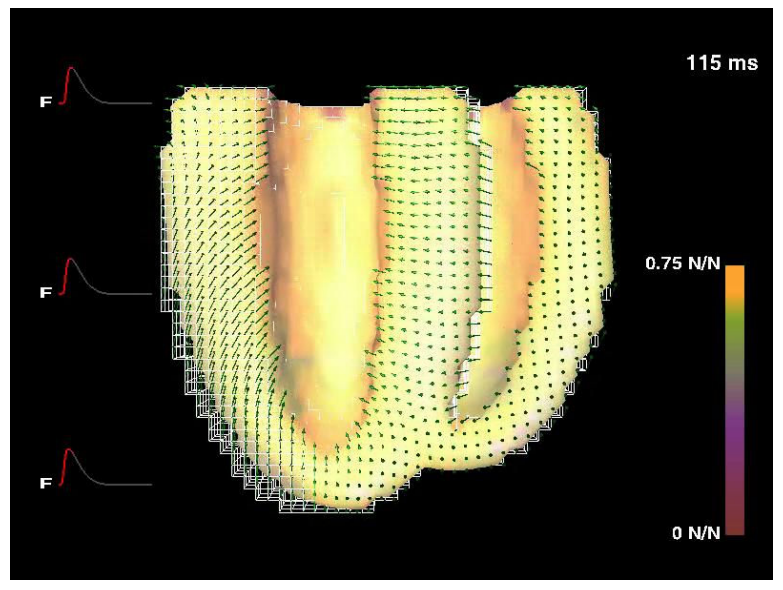

(b)

Figure 1. Results of simulation with analytical biventricular model. (a) Full and (b) halved model are shown at time of maximal developed tension. The course of developed tension at apical, equatorial, and basal midwall positions is depicted on the left side. Arrows indicate displacements.

model led to a significantly smaller rotation of the apex and its movement off basis.

The simulations were demanding concerning computing resources and thus performed on a Silicon Graphics Origin 3000 compute server with 32 GB of main memory and 64 processors of type R14000/600 MHz. In both simulations 32 processors were employed, each for $\approx 7 d$.

\section{Conclusions}

The hybrid models were applied to illustrate the interplay between the different basic models and the importance of appropriate anatomical modeling. The results of simulations, i.e. patterns of electrical propagation, developed tension, and mechanical deformation, were analyzed. The models show similarities of the spread

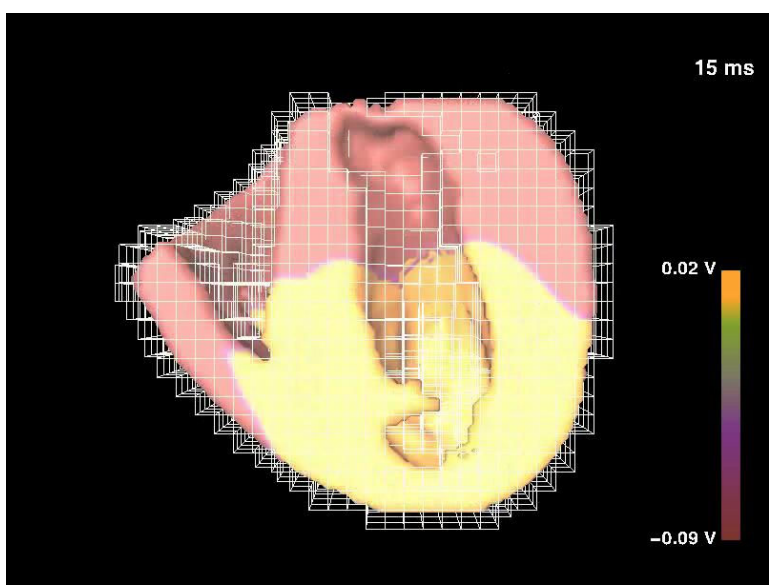

(a)

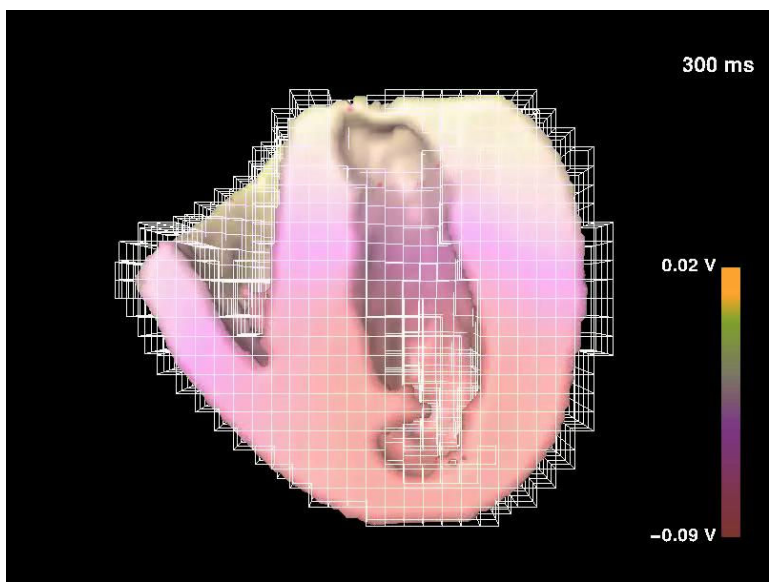

(b)

Figure 2. Results of simulation with MR DTI biventricular model. Several aspects of electrophysiology and mechanics in halved model are illustrated. The distribution of transmembrane voltage $V_{m}$ is shown colorcoded at time (a) 15 and (b) $300 \mathrm{~ms}$ after application of stimulus.

of excitation, but significant differences concerning deformation. The MR DTI model led to an uncharacteristic deformation, which may result from the fixation of the canine heart.

The presented simulations gave insights for further development of realistic models of the whole heart, e.g. for studying cardiac arrhythmias and for the computer aided planning of surgical interventions. Future work will profit from recent advances of MR DTI, which offer in-vitro measurement and increased signal to noise ratio.

\section{Acknowledgments}

This work has been supported by the Richard A. and Nora Eccles Fund for Cardiovascular Research and awards from the Nora Eccles Treadwell Foundation. 


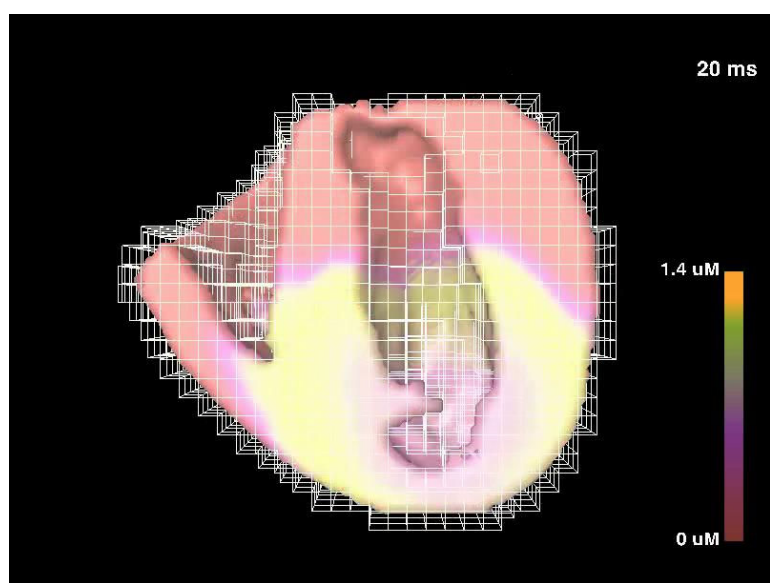

(a)

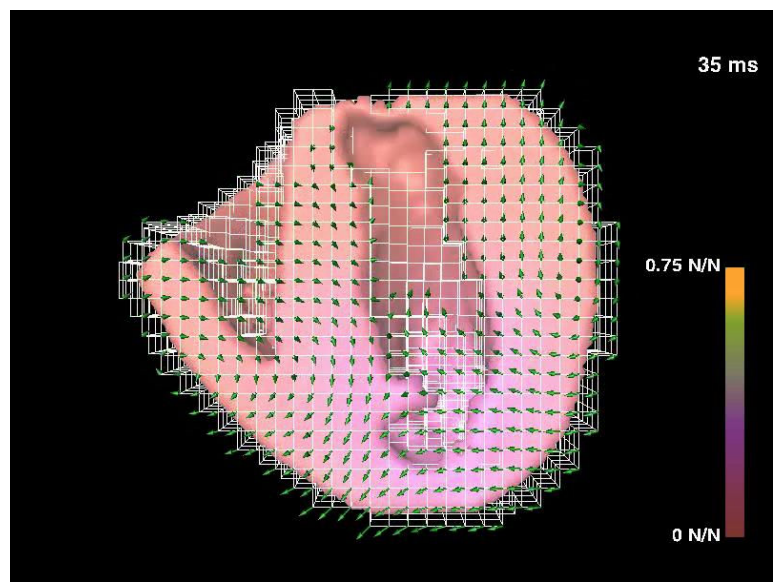

(b)

Figure 3. Results of simulation with MR DTI biventricular model with (a) the distribution of intracellular calcium $\left[\mathrm{Ca}^{2+}\right]_{i}$ at time $20 \mathrm{~ms}$, and (b) tension developed at 35 $m s$, i.e. an initial phase of contraction.

The authors gratefully acknowledge computing resources kindly provided by the Scientific Computing and Imaging Institute and the NIH NCRR Center for Bioelectric Field Modeling, Simulation, and Visualization.

\section{References}

[1] Streeter jr. DD, Bassett DL. An engineering analysis of myocardial fiber orientation in pig's left ventricle in systole. Anatomical Record 1966;155:503-512.

[2] Sachse FB, Henriquez C, Seemann G, Riedel C, Werner $\mathrm{CD}$, Penland RC, Davis B, Hsu E. Modeling of fiber orientation in the ventricular myocardium with MR diffusion imaging. In Proc. Computers in Cardiology, volume 28. Sep. 2001; 617-620.

[3] Gonzalez RC, Woods RE. Digital Image Processing. Reading, Massachusetts; Menlo Park, California: AddisonWesley, 1992. ISBN 0-201-60078-1.
[4] Zerfass P, Sachse FB, Werner CD, Dössel O. Deformation of surface nets for interactive segmentation of tomographic data. In Biomedizinische Technik, volume 45-1. Sep. 2000; 483-484.

[5] Noble D, Varghese A, Kohl P, Noble P. Improved guineapig ventricular cell model incorporating a diadic space, $I_{K r}$ and $I_{K s}$, and length- and tension-dependend processes. Can J Cardiol Jan. 1998;14(1):123-134.

[6] Sachse FB. Computational Cardiology: Modeling of Anatomy, Electrophysiology, and Mechanics. LNCS 2966. Berlin, Heidelberg, New York: Springer, 2004.

[7] Henriquez CS, Muzikant AL, Smoak CK. Anisotropy, fiber curvature and bath loading effects on activation in thin and thick cardiac tissue preparations: Simulations in a threedimensional bidomain model. J Cardiovasc Electrophysiol May 1996;7(5):424-444.

[8] Glänzel K. Kraftentwicklung im Sarkomer unter Berücksichtigung elektromechanischer Kopplung. Diploma Thesis, Institut für Biomedizinische Technik, Universität Karlsruhe (TH), Sep. 2002.

[9] Sachse FB, Glänzel K, Seemann G. Modeling of protein interactions involved in cardiac tension development. Int $\mathbf{J}$ Bifurc Chaos 2003;13(12):3561-3578.

[10] Rice JJ, Winslow RL, Hunter WC. Comparison of putative cooperative mechanisms in cardiac muscle: Length dependence and dynamic responses. Am J Physiol Circ Heart 1999;276:H1734-H1754.

[11] Gordon AM, Regnier M, Homsher E. Skeletal and cardiac muscle contractile activation: Tropomyosin "rocks and rolls". News Physiol Sci 2001;16:49-55.

[12] Bers DM. Excitation-Contraction Coupling and Cardiac Contractile Force. Dordrecht, Netherlands: Kluwer Academic Publishers, 1991. ISBN 0-7923-1186-8.

[13] Spudich JA. TIMELINE: The myosin swinging crossbridge model. Nature Reviews Molecular Cell Biology 2001;2:387-392.

[14] Mohr MB, Blümcke LG, Seemann G, Sachse FB, Dössel O. Volume modeling of myocard deformation using a spring mass system. LNCS 2673 2003;332-339.

[15] Kuhn C, Kühnapfel U, Deussen O. Echtzeitsimulation deformierbarer Objekte zur Ausbildungsunterstztung in der minimal-invasiven Chirurgie. In Proc. Intl. GI Workshop: Modelling, Virtual Worlds, Distributed Graphics. Infix, 1995;

[16] Bathe KJ. Finite Element Procedures. Upper Saddle River, New Jersey: Prentice Hall, 1996. ISBN 0-13-301458-4.

[17] Guccione JM, McCulloch AD, Waldman LK. Passive material properties of intact ventricular myocardium determined from a cylindrical model. J Biomechanical Engineering Feb. 1991;113:42-55.

Address for correspondence:

PD Dr.-Ing. F. B. Sachse

Nora Eccles Harrison Cardiovascular Research and Training Institute

University of Utah, Salt Lake City, Utah, USA

E-mail: fs@cvrti.utah.edu 